\title{
ESTUDIO DEL CRECIMIENTO Y DESARROLLO DE NIÑOS ECUATORIANOS RESIDENTES EN QUITO, SEGÚN SU ORIGEN SOCIAL
}

\author{
Autores: \\ Claude Monnier ${ }^{1}$ \\ Roland Hauspie ${ }^{1}$ \\ Juan Cruz Albornoz ${ }^{2}$
}

Facultad de Turismo, Universidad Tecnológica Equinoccial, Rumipamba y Bourgeois, Apartado 17-01-2764, Quito - Ecuador jcruz@ute.edu.ec 



\section{Resumen}

El documento que presentamos es una relación Histórica, Científica y Socio demográfica de la investigación que se llevó a cabo a través de Tres Misiones Científicas realizadas en los años 1999, 2007 y 2009, con la participación de las Universidades ULB y VUB de Bruselas, Bélgica y la Universidad Tecnológica Equinoccial, de Quito - Ecuador.

La propuesta original y la dirección del Proyecto de investigación correspondió al Laboratorio de Antropología Física y Genética Humana de la Universidad Libre de Bruselas; la organización local, convenios institucionales para la aprobación de encuestas socio demográficas y captura de las muestras biométricas correspondió a la Facultad de Turismo y Conservación Ambiental de la Universidad Tecnológica Equinoccial. La participación de cada centro se detalla en el informe que presentamos.

El contenido de este documento describe en primer lugar, el análisis realizado de las muestras de las 9 medidas corporales según F. Twiesselmann y de las encuestas socio - demográficas que las determinan.

Las muestras y encuestas fueron tomadas en establecimientos públicos y privados de la ciudad de Quito.

Presentamos a continuación los resultados obtenidos, tanto en el área socio demográfica como biométrica.

Por último se podrán valorar cuadros que describen las curvas de crecimiento y las tallas de varones y mujeres, peso medio de los jóvenes varones y mujeres y de $\mathrm{IMC} / \mathrm{Kg} / \mathrm{m}^{3}$, igualmente de los jóvenes varones y mujeres de la ciudad de Quito. 


\section{Abstrac}

The document presented is a historical scientific relationship and demographic partner in the research carried out by three scientific missions approved out in 1999, 2007 and 2009, with the participation of the universities ULB and VUB Brussels, Belgium and the Universidad Tecnológica Equinoccial, of Quito - Ecuador.

The original proposal and the direction of the research project corresponded to the laboratory of physical anthropology and human genetics at the free University of Brussels; the local organization, institutional agreements for approval of surveys capture of biometric samples and demographic partner corresponded to the Faculty of tourism and environmental conservation of the Universidad Tecnológica Equinoccial. The participation of each center is detailed in the report we present.

The contents of this document describes in the first place, the analysis of samples of bodily according to Twiesselmann measures 9 and polls socio - demographic that determine them.

Samples and surveys were taken in public and private of the Quito City establishments.

Here are the results obtained, both in the partner area demographic as biometric.

Tables that describe the growth curves and carvings of males and females, average weight of young men and women and IMC/Kg/m², also young men and women of the city of Quito may finally be assessed. 


\section{Introducción}

Es difícil avanzar una cifra precisa del número de personas que viven en el mundo de forma permanente a alturas superiores a 2500 metros del nivel del mar. Estas personas se encuentran tanto en Africa, en Asia como en América; y se concentran de preferencia, en los Andes, en el Himalaya, en las altas tierras de Etiopía o en Asia central constituyendo un ejemplo de la adaptación biológica del hombre a su medio ambiente.

Estos grupos humanos han sido objeto de numerosas investigaciones, tanto en antropología (Eveleth et al., 1976; Frisancho, 1975; Dieu-Cambrezy et al., 1993), como en fisiología o sobre su estado de salud (Niermeyer et al., 2008). En alta montaña, el organismo está confrontado a toda una serie de factores climáticos tales como el frío, la violencia del viento, la intensidad de la radiación solar, la baja del grado higrométrico y sobre todo la hipoxia que es la consecuencia de la disminución parcial de la presión en oxígeno del aire (Benoist, 1980).

Las áreas pobladas a alta altitud, presentan no solamente un entorno físico particular, sino que igualmente elementos de asentamientos ancestrales, de condiciones socio-económicas difíciles y un acceso restringido a los centros de salud.

En lo que respecta al desarrollo físico de niños y adolescentes, tema de este trabajo, variadas observaciones han sido ya realizadas. Ellas hacen hincapié en el hecho de que en general hay una disminución de la estatura con la altitud (Crespo et al., 1995; Bejarano et al., 2009). Este retraso en el crecimiento de los individuos comenzaría desde el nacimiento (Leonard et al., 1995), sería congénito (vida fetal) (Bennet et al., 2008) y por tanto de origen hereditario (Moore, 2001) y sería una respuesta del organismo al estrés causado por la disminución de la concentración de oxígeno en la sangre (hipoxemia) (Facchini, 2003)
También ha sido avanzado, que el retraso del desarrollo físico, sería más bien una consecuencia de la mala nutrición que a un efecto de la altitud (Harris et al., 2001). Esta última hipótesis ha sido propuesta, sobre todo, para las poblaciones de las zonas del Himalaya y no Andinas. O bien los factores económicos contribuirían a acentuar las diferencias (Stinson, 2009; Pawson et al., 2001).

Se ha proseguido el estudio del crecimiento iniciado en 1999 (Monnier et al., 2004; 2009; Vercauteren et al., 2009) de niños viviendo en medio urbano y que habitan a una altitud que oscila entre 2800 y 3100 metros sobre el nivel del Mar. El universo urbano siendo heterogéneo, este estudio ha sido llevado a cabo tomando en cuenta el medio ambiente socio-económico de los individuos encuestados. Estos últimos han sido clasificados en tres categorías sociales: baja, media y alta.

\section{Material y método.}

Ecuador es un País que se encuentra al noroeste de América del sur. Limita por el noreste con Colombia, por el este y el sur con Perú y por el oeste con el océano Pacífico. Su territorio está dividido en cuatro regiones geográficas naturales, de las cuales tres se sitúan sobre el continente y la restante es el archipiélago de las Galápagos en el Pacífico.

La Costa comprende una llanura litoral y una cadena montañosa de pequeña altura (800 metros). Esta región natural se extiende a lo largo de todo el país siguiendo el curso del Océano Pacífico, teniendo como límite, al este la cordillera de los Andes; esta región concentra casi el 30 \% de la población del país. La Sierra (o altas tierras centrales) agrupa 60\% de la población del país que vive a una altitud media superior a los 3000 metros. El Oriente constituye la región amazónica del Ecuador. A pesar que esta región ocupa más de la mitad del territorio ecuatoriano, su población no alcanza al $10 \%$ de los habitantes del país. Finalmente, el país comprende igualmente, el archipiélago de las Galápagos situado en el Océano Pacífico. 
Quito, la capital del Ecuador, cuenta actualmente con alrededor de 2 millones de habitantes y constituye el principal polo de desarrollo económico de la Sierra.

Este estudio abarca un total de 3034 sujetos repartidos de la manera siguiente: $50,6 \%$ de varones (niños) y $49,4 \%$ de mujeres (niñas). La Tabla 1 muestra, en valor absoluto, las observaciones según el género y el año de la encuesta.

\begin{tabular}{|l|l|l|l|}
\hline & $\begin{array}{l}\text { Encuesta } \\
1999\end{array}$ & $\begin{array}{l}\text { Encuesta } \\
2007 / 09\end{array}$ & Total \\
\hline Niños & 459 & 1075 & 1534 \\
\hline Niñas & 629 & 871 & 1500 \\
\hline Total & 1088 & 1946 & 3034 \\
\hline
\end{tabular}

Tabla 1: Repartición de efectivos encuestados según el género y el año de la observación

\section{Elaborado por los investigadores}

Las mensuraciones corporales retenidas y que responden de mejor manera a un equilibrio entre la relación peso/estatura, la obesidad y el desarrollo muscular y adiposo son las siguientes: mensuración del peso, de la estatura, de la estatura sentada, del diámetro biacromial, del diámetro bicrestal, perímetro del brazo extendido, perímetro del brazo en flexión, perímetro del muslo y diámetro toráxico transversal. Estas mensuraciones han sido el resultado de tres misiones precedentes. Para la elaboración de las curvas de crecimiento, los datos han sido tratados por el método de LMS, que es actualmente el más utilizado para la estimación de los percentiles. Las diferentes mensuraciones y dimensiones corporales han sido compiladas según la técnica biométrica propuesta por la OMS (WHO, 1995). La estatura y el BMI han sido convertidos en z-scores según las curvas de referencias de la OMS (WHO, 2006). Las tasas de sobrepeso y sub-peso han sido establecidas de acuerdo a los criterios instituidos por Cole (2000; 2007).

Además de las mensuraciones antropométricas, ciertos antecedentes socio-demográficos han sido recopilados en relación a los niños y a sus padres. Los parámetros retenidos son los lugares de nacimiento, las profesiones y los niveles de estudio de los padres, de una parte, y de otra parte para los sujetos encuestados el lugar y fecha de nacimiento, el rango social y la dimensión de la fratría al momento que la información fue recogida. La ascendencia social de los niños ha sido establecida teniendo en cuenta el tipo de escuela frecuentado. En este trabajo han sido retenidos solamente los lugares de nacimiento de los sujetos y de sus progenitores y los niveles de estudio de estos últimos.

Acompañando estos datos socio-económicos, un tercer grupo de preguntas dirigidas a conocer las costumbres alimenticias ha sido propuesto. La encuesta sobre la alimentación ha sido llevada a cabo siguiendo el método recomendado por Truswell(1987) como también por los nutricionistas (Apfelbaun et al., 2004). Este método consiste en que el sujeto encuestado, se recuerde de todo lo que él ha comido en el curso de las últimas 24 horas anteriores a la entrevista.

\section{Perfil socio-demográfico poblacional.}

El origen geográfico de los niños se estableció teniendo en cuenta el lugar de nacimiento. No todos los cuestionarios recibidos hacían mención del origen nativo de los encuestados; solamente 2579 cuestionarios citaban esta información, es decir, un 85\% de los sujetos interrogados. La Tabla 2, muestra la repartición de los sujetos según su lugar de nacimiento y su clase social.

\begin{tabular}{|l|l|l|l|l|l|l|}
\hline \multicolumn{2}{|c|}{ Categorías sociales } & \multicolumn{2}{c|}{ Baja } & \multicolumn{2}{c|}{ Media } & \multicolumn{2}{c|}{ Alta } \\
\hline & Efectivos & $\%$ & Efectivos & $\%$ & Efectivos & $\%$ \\
\hline Quito & 487 & 75,7 & 1628 & 90,0 & 113 & 89,7 \\
\hline Costa & 41 & 6,3 & 40 & 2,2 & 2 & 1,6 \\
\hline Sierra & 103 & 16,3 & 119 & 6,6 & 5 & 4,0 \\
\hline A mazona & 4 & 0,6 & 9 & 0,5 & - & - \\
\hline Extranjero & 7 & 1,1 & 13 & 0,7 & 6 & 4,7 \\
\hline Total & 644 & 100,0 & 1809 & 100,0 & 126 & 100,0 \\
\hline
\end{tabular}

Tabla 2 : Lugar de nacimiento de los sujetos estudiados según las categorías sociales a las cuales pertenecen 
Poca diferencia es observada, según su origen geográfico, entre los niños de categorías sociales mediana y alta; ellos provienen principalmente de Quito y de la región de la Sierra. Por el contrario, en los niños de la tercera categoría social, se encuentra una proporción mínima de niños oriundos de Quito. Alrededor de 75\% contra 90\% para los niños de las otras dos categorías sociales.

Cuando se totaliza en los tres grupos sociales los niños nacidos en alta altitud (Quito y la Sierra) se registran porcentajes del mismo orden de magnitud. Ellos agrupan entre $92 \%$ y $96 \%$ de los sujetos.

El origen geográfico de los progenitores figura respectivamente para los padres (Tabla 3) y para las madres (Tabla 4). Las observaciones engloban un $86,5 \%$ de sujetos para los padres y $89,3 \%$ para las madres. En el seno de la misma categoría social, las distribuciones geográficas de hombres y mujeres son similares. Por el contrario, de una categoría social a otra se registran diferencias. Es así como la proporción de nativos de la ciudad de Quito es similar tanto para los hombres y mujeres de clase media y alta, oscilando alrededor del 60\% de los efectivos; a su vez, entre los padres de clase modesta el número de efectivos es apenas superior al tercio.

Como Quito, capital del país, es además la principal ciudad de la Sierra, ella beneficia a la inmigración proveniente mayoritariamente de la Sierra. Esta inmigración alimenta principalmente los efectivos de la categoría social baja y en una mínima proporción la clase media.

\begin{tabular}{|c|c|c|c|c|c|c|}
\hline \multicolumn{2}{|c|}{ Categorías sociales } & \multirow{2}{*}{$\begin{array}{l}\text { Bajas } \\
\%\end{array}$} & \multicolumn{2}{|l|}{ Medias } & \multicolumn{2}{|l|}{ Altas } \\
\hline & Efectivos & & Efectivos & $\%$ & Efectivos & $\%$ \\
\hline Quito & 213 & 36,8 & 1054 & 57,7 & 77 & 36,6 \\
\hline Costa & 78 & 13,4 & 98 & 5,5 & 10 & 8,3 \\
\hline Sierra & 265 & 46,4 & 649 & 35,5 & 22 & 18,2 \\
\hline A mazona & 5 & 0,5 & 11 & 0,6 & - & - \\
\hline Extranjero & 17 & 2,9 & 14 & 0,7 & 12 & 9,9 \\
\hline Total & 578 & 100,0 & 1826 & 100,0 & 121 & 100,0 \\
\hline
\end{tabular}

Tabla 3: Orígenes geográficos de los padres según su categoría social
A excepción de la Costa, las otras regiones naturales del país no aportan más que una débil corriente migratoria hacia la capital. La inmigración internacional, tanto en relación a hombres como a mujeres, es muy modesta y concierne sobre todo a personas nativas de países Andinos (Perú, Chile, Colombia) y atañe generalmente individuos de clase acomodada.

\begin{tabular}{|l|l|l|l|c|l|l|}
\hline \multicolumn{2}{|l|}{ Categorías sociales Baja } & \multicolumn{2}{c|}{ Media } & \multicolumn{2}{c|}{ Alta } \\
\hline & Efectivos & $\%$ & Efectivos & $\%$ & Efectivos & $\%$ \\
\hline Quito & 210 & 35,0 & 1157 & 58,1 & 72 & 59,5 \\
\hline Costa & 90 & 15,0 & 157 & 7,9 & 13 & 10,7 \\
\hline Sierra & 274 & 45,8 & 629 & 31,6 & 24 & 19,9 \\
\hline A mazona & 8 & 1,3 & 23 & 1,2 & - & - \\
\hline Extranjero & 17 & 2,8 & 24 & 1,2 & 12 & 9,9 \\
\hline Total & 599 & 100,0 & 1990 & 100,0 & 121 & 100,0 \\
\hline
\end{tabular}

Tabla 4: Orígenes geográficos de las madres según su categoría social

Finalmente, más del $92 \%$ de los niños han nacido en zonas montañosas y para los padres este porcentaje es superior al $80 \%$ de los efectivos. De un interés casi exclusivamente andino, la Capital se abre hacia la Costa e incluso hacia el extranjero.

El nivel de estudio alcanzado por los progenitores y, particularmente en el caso del padre, es revelador de la clase social a la cual pertenece. Los resultados se refieren a solamente el 79;5\% de los padres y al $83,4 \%$ de las madres y son representados en la Tabla 5 para los padres y para las madres en la Tabla 6. Se percibe en las categorías sociales estudiadas una forma de endogamia al nivel de la escolaridad. 


\begin{tabular}{|l|l|c|l|l|l|l|}
\hline \multicolumn{2}{|c|}{ Categorías sociales Baja } & \multicolumn{2}{|c|}{ Media } & \multicolumn{2}{c|}{ Alta } \\
\hline & Efectivos & $\%$ & Efectivos & $\%$ & Efectivos & $\%$ \\
\hline Primaria & 330 & 53,7 & 163 & 9,7 & - & - \\
\hline Sec. inf. & 190 & 30,9 & 271 & 16,2 & 4 & 3,4 \\
\hline Sec. sup. & 55 & 8,9 & 510 & 30,4 & 5 & 4,2 \\
\hline Sup. n.Univ. & 22 & 3,8 & 579 & 34,5 & 50 & 12,0 \\
\hline Universitario & 17 & 2,8 & 155 & 9,2 & 60 & 50,4 \\
\hline Total & 614 & 100,0 & 1678 & 100,0 & 119 & 100,0 \\
\hline
\end{tabular}

Tabla 5: Clasificación de los padres de acuerdo a su nivel de estudio y categoría social

La clasificación de los padres de acuerdo al nivel de estudio alcanzado, corresponde a su jerarquía social. La más importante proporción de personas que poseen un diploma ya sea de nivel primario, o de nivel secundario inferior, se encuentran en la categoría social más baja. Por el contrario, la más fuerte proporción de diplomados universitarios se concentra entre los miembros de la clase social más alta. Los miembros de la clase media son, en su gran mayoría, titulares de diplomas de nivel intermediarios.

\begin{tabular}{|l|l|l|l|l|l|l|}
\hline \multicolumn{2}{|c|}{ Categorías sociales Baja } & \multicolumn{3}{c|}{ Media } & \multicolumn{2}{c|}{ Alta } \\
\hline & Efectivos & $\%$ & Efectivos & $\%$ & Efectivos & $\%$ \\
\hline Primaria & 376 & 58,3 & 178 & 10,1 & 1 & 0,8 \\
\hline Sec. inf. & 190 & 29,5 & 286 & 16,2 & 4 & 3,4 \\
\hline Sec.sup. & 46 & 7,6 & 588 & 33,3 & 10 & 8,4 \\
\hline Sup. n. Univ. & 22 & 3,4 & 539 & 30,5 & 48 & 40,3 \\
\hline Universitaria & 8 & 1,2 & 175 & 9,9 & 56 & 47,1 \\
\hline Total & 645 & 100,0 & 1766 & 100,0 & 119 & 100,0 \\
\hline
\end{tabular}

Tabla 6: Clasificación de las madres de acuerdo a su nivel de estudio y categoría social

\section{Los datos}

La Tabla 7 resume el número de observaciones realizadas en el curso de las misiones de 1999 y 2007/2009.

Las mensuraciones han sido llevadas a cabo, exclusivamente, en establecimientos escolares situados en la ciudad de Quito y su aglomeración. Esencialmente concierne a niños que viven en medio urbano. Como los datos han sido recolectados en períodos diferentes, las observaciones no cubren, de acuerdo a las categorías, la totalidad del dominio de edades estudiado.

Los niños incluidos en la categoría social baja tienen edades comprendidas entre 11/12 años y 19/20 años; los de la clase media oscilan entre 4 años y 18/19 años y los de categoría social alta entre 5 años y 14/15 años. De esta manera la evolución secular no podrá ser calculada para los niños de la clase media que han sido mensurados en 1999 y en 2007.

\begin{tabular}{|c|c|c|c|}
\hline Edad & Niños & Niñas & Total \\
\hline 4 & 4 & 7 & 11 \\
\hline 5 & 37 & 27 & 64 \\
\hline 6 & 99 & 96 & 195 \\
\hline 7 & 83 & 99 & 182 \\
\hline 8 & 85 & 126 & 211 \\
\hline 9 & 72 & 98 & 170 \\
\hline 10 & 102 & 110 & 212 \\
\hline 11 & 118 & 79 & 197 \\
\hline 12 & 181 & 132 & 313 \\
\hline 13 & 170 & 118 & 288 \\
\hline 14 & 157 & 148 & 305 \\
\hline 15 & 157 & 127 & 284 \\
\hline 16 & 99 & 115 & 214 \\
\hline 17 & 103 & 118 & 221 \\
\hline 18 & 37 & 64 & 101 \\
\hline 19 & 13 & 22 & 35 \\
\hline $20 y+$ & 17 & 14 & 31 \\
\hline
\end{tabular}

Tabla 7: Observaciones realizadas en función de la edad y sexo 


\section{Resultados}

\section{Estatura y BMI}

Según las categorías sociales, las estaturas y el BMI de los niños y de las niñas han sido convertidos en z-scores de acuerdo a las indicaciones de la OMS. Los resultados obtenidos para los niños son presentados en la figura 1 y para las niñas en la figura 2. Comparados a las normas de la OMS, los z-scores de la estatura de los niños es $-0,87 \pm 1,01$ y el de las niñas es $-1,13 \pm 0,98$. La estatura de los niños es superior al de las niñas.

Figura 1
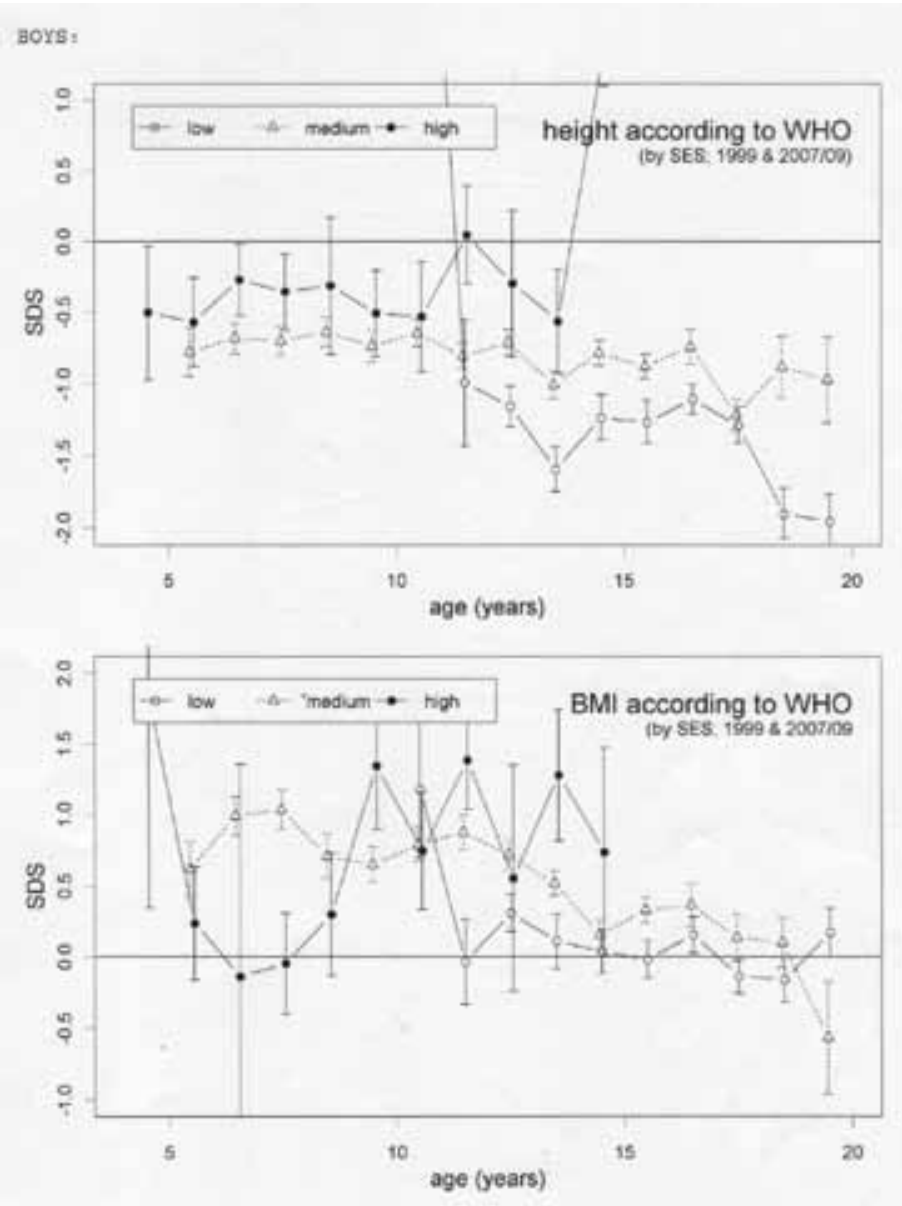

Elaborado por los investigadores
Figura 2:
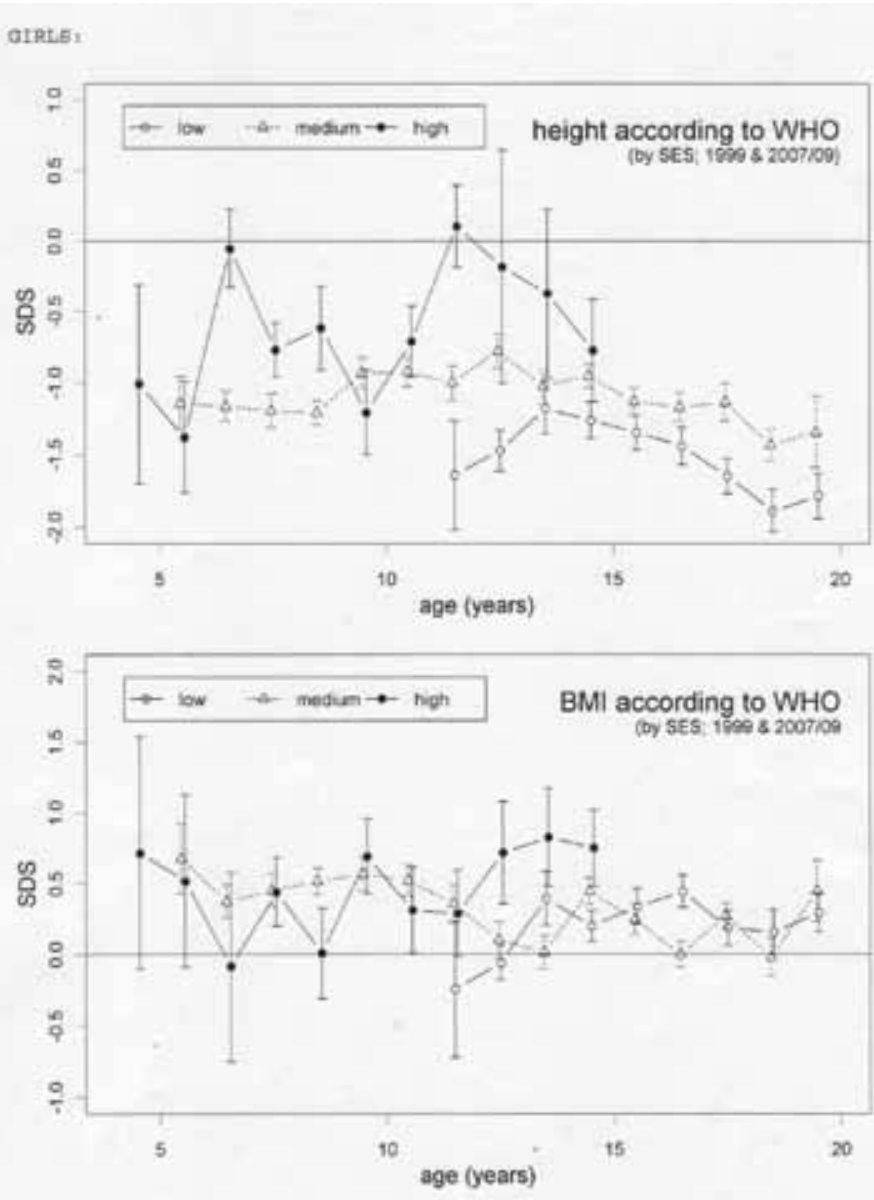

Elaborado por los investigadores

Las estaturas tanto de los niños como de las niñas han sido comparadas a los valores de referencia de la OMS. Independientemente de la categoría social, las estaturas de los niños y niñas son notoriamente inferiores a las normas de la OMS.

Las poblaciones urbanas no son homogéneas y se registran diferencias de estaturas entre los niños según su origen social. Las estaturas más bajas predominan en la categoría social la más modesta y los individuos más altos se encuentran en la categoría social más pudiente. Los resultados intermediarios se observan entre los niños de la clase media. 
Si las estaturas de los niños de Quito, independientemente de sus clases sociales, son notoriamente inferiores a las normas de la OMS, el BMI es netamente superior a las normas de la OMS. Sin embargo, el BMI de los niños de diferentes clases sociales no se distingue los unos de los otros, en oposición a los resultados relativos a las estaturas.

\section{Sobrepeso y déficit ponderal}

Las categorías de sobrepeso y de déficit ponderal han sido establecidas por Cole $(2000 ; 2007)$ y los resultados figuran para los niños en la Tabla 8 y para las niñas en la Tabla 9.

Una fracción importante de sujetos pertenecientes a medios urbanos, sufren de sobrepeso e incluso de obesidad, esta característica siendo más marcada en el caso de los varones. Es entre las clases sociales más desfavorecidas que se registra la más baja proporción de sujetos aquejados de sobrepeso. Esta constatación es sobre todo válida para los varones.

Tabla 8: Sobrepeso y déficit ponderal para los varones según su clase social

\begin{tabular}{|l|l|l|l|l|l|l|}
\hline & Baja & $\%$ & Mediana & $\%$ & Alta & $\%$ \\
\hline-3 & 1 & 0,3 & 6 & 0,5 & 2 & 2,4 \\
\hline-2 & 2 & 0,6 & 6 & 0,5 & 2 & 2,4 \\
\hline-1 & 15 & 4,4 & 31 & 2,8 & 2 & 2,4 \\
\hline 0 & 279 & 81,1 & 795 & 71,8 & 55 & 66,3 \\
\hline 1 & $43^{* * *}$ & 12,5 & 208 & 18,8 & 15 & 18,1 \\
\hline 2 & 4 & 1,2 & 61 & 5,5 & 7 & 8,4 \\
\hline
\end{tabular}

${ }^{*} \mathrm{p}<0,05:{ }^{* *} \mathrm{p}<0,01: * * * \mathrm{p}<0,001$

Elaborado por los investigadores

Los tests han sido efectuados teniendo como referencia los resultados obtenidos para los niños de clase media. De la lectura de estos resultados se puede deducir que no hay una diferencia significativa entre los niños de clase media y alta en función, ya sea, del sobrepeso o del déficit ponderal. Por el contrario, las diferencias son bien marcadas entre los niños de clase modesta y las otras categorías sociales. Más de un cuarto de los varones de las categorías sociales media y alta sufren de sobrepeso, mientras que esta proporción cae a 13,8\% entre los niños de categoría social baja.

Tabla 9: Sobrepeso y déficit ponderal para las niñas según su clase social.

\begin{tabular}{|l|l|l|l|l|l|l|}
\hline & Baja & \% & Media & \% & Alta & $\%$ \\
\hline-3 & - & - & 3 & 0,3 & 2 & 2,2 \\
\hline-2 & 4 & 1,1 & 12 & 1,1 & 2 & 2,2 \\
\hline-1 & 23 & 6,6 & 55 & 5,2 & 7 & 7,5 \\
\hline 0 & 266 & 75,8 & 786 & 74,4 & 65 & 69,9 \\
\hline 1 & 53 & 15,1 & 179 & 17,0 & 14 & 15,1 \\
\hline 2 & 5 & 1,4 & 21 & 2,0 & 3 & 3,2 \\
\hline
\end{tabular}

$* \mathrm{p}<0,05: * * \mathrm{p}<0,01: * * * \mathrm{p}<0,001$

Elaborado por los investigadores

Aparentemente, no habría ninguna diferencia significativa entre las niñas que sufren de sobrepeso o de déficit ponderal, según las diferentes clases sociales. La proporción de niñas aquejadas de sobrepeso oscila entre $16,5 \%$ y $19 \%$

\section{Costumbres alimenticias.}

Buscando establecer una relación eventual entre el desarrollo corporal de los niños y sus costumbres alimenticias, se ha solicitado a cada individuo de enumerar la totalidad de los alimentos consumidos en el curso de las 24 horas que preceden la encuesta. Las informaciones son cualitativas y permiten sugerir, eventualmente, de elementos explicativos de las diferencias detectadas en el desarrollo físico de los individuos según su clase social. El análisis de los resultados se realizó adoptando como referencia el consumo de los individuos de la clase media. Los resultados son presentados según el número promedio de veces que el producto, en cuestión, es citado. A este valor promedio, se agrega el porcentaje de sujetos que han formulado una respuesta para el producto estudiado. Los tests de significación han sido establecidos en relación a las repuestas formuladas por los individuos de la clase media. 
Tabla 10: Consumo alimentario según las categorías sociales. (la referencia retenida es la clase media)

\begin{tabular}{|l|l|l|l|l|l|l|}
\hline \multicolumn{2}{|c|}{ Categorías sociales Baja } & \multicolumn{2}{c|}{ M ediana } & \multicolumn{1}{c|}{ Alta } \\
\hline & n. media & $\%$ & n. media & $\%$ & n. media & $\%$ \\
\hline Prod.lacteos & $0,70^{* * *}$ & 50,8 & 1,06 & 69,2 & $1,84^{* * *}$ & 87,9 \\
\hline Prod. Carnes & $1,41^{* *}$ & 80,9 & 1,22 & 71,4 & $1,79^{* * *}$ & 71,6 \\
\hline Prod. Legumbre & $0,45^{* *}$ & 35,8 & 0,34 & 27,1 & $0,52^{* *}$ & 40,5 \\
\hline $\begin{array}{l}\text { B ebidas } \\
\text { azucaradas }\end{array}$ & $1,51^{* * *}$ & 84,1 & 2,30 & 95,6 & $1,76^{* * *}$ & 89,7 \\
\hline $\begin{array}{l}\text { Prod. } \\
\text { azucarad.salados }\end{array}$ & $0,17^{* * *}$ & 14,7 & 0,08 & 6,3 & 0,11 & 9,5 \\
\hline Fritas & $0,14^{* * *}$ & 14,3 & 0,03 & 3,2 & 0,00 & - \\
\hline
\end{tabular}

${ }^{*} \mathrm{p}<0,05: * * \mathrm{p}<0,01: * * * \mathrm{p}<0,001$

Elaborado por los investigadores

La interpretación debe ser hecha en función del comportamiento de los individuos de la clase media. Los productos tales como el arroz, el maíz, las patatas y la mandioca, no han sido retenidos en este estudio por cuanto ellos constituyen la dieta básica ecuatoriana y se consumen casi todos los días, bajo la forma de sopas, independientemente de la clase social a la cual pertenece el individuo.

Los resultados muestran una notable diferencia según la clase social a la cual pertenecen los niños y adolescentes encuestados. (Tabla 10).

Si los comportamientos alimentarios difieren profundamente de una categoría social a otra, se pueden poner en evidencia ciertos rasgos o elementos que permiten analizar los resultados obtenidos. Es así como los niños de la categoría social acomodada consumen en promedio 2,5 veces más leche o productos lácteos que los niños de clases modestas. Por el contrario, los niños de la clase media consumen 1,5 veces más bebidas azucaradas que aquellos de clase modesta; e igualmente se constata una afición marcada por las papas fritas de los niños que provienen de clases modestas. Estos productos parecen ser ignorados o poco consumidos por los niños de las otras clases sociales.
El consumo de productos cárnicos parece ser bastante importante. Frecuentemente mencionados por las clases acomodadas, son menos citados por los individuos de las clases medias que consumirían menos carne que la clase baja.

\section{Discusión}

Los resultados obtenidos para la talla de los niños, muestran claramente que durante todo el período de crecimiento, aquellos que viven en altura, en particular los de Quito, presentan una talla inferior a las normas de la OMS. Estos resultados no divergen de aquellos descritos en diferentes regiones de gran altitud. Las tallas reducidas al final del desarrollo, como también en el curso del mismo, son observados en diferentes lugares y puestos en evidencia por diferentes autores (Dieu-Cambrezy et al., 1993; Leonard et al., 1995; Bejaro et al., 2009).

Frecuentemente, las observaciones se han centralizado sobre etnias bien precisas como en Bolivia, con los Aymaras (Stinson, 1980; Greksa et al.,1984; Kim et al., 1991) o los Quechuas o los Mesticos (Kim et al.,1991), en Perú son las etnias Quechuas (Pawson et al., 2001) y Aymaras ( de Meer et al., 1993), en Chile los Aymaras (Dittmar , 1997), pero relativamente poco sobre las poblaciones mestizas que viven en altitud y en medio urbano. Como el medio urbano no es homogéneo; las estratificaciones sociales son más profundas en América latina que en otros lugares del planeta (Klein et al., 2005).

El crecimiento un poco más lento se prolongaría, en promedio de 1 a 2 años más con respecto a jóvenes que viven al nivel del mar (Benoist, 1980). Las tallas más pequeñas de los niños que viven a grandes altitudes, son a menudo interpretadas como una respuesta de adaptación al estrés causado por la hipoxia (Frisancho, 1975). Hay que reconocer que otros factores pueden matizar la interpretación de estos resultados: las variaciones del desarrollo in utero ( Illsley et 
al., 2010), las enfermedades, el régimen alimenticio y las condiciones socio-económicas y sanitarias.( Dieu-Cambrezy et al., 1993).

Los resultados obtenidos para diversas poblaciones amerindias (Aymará de Bolivia, Quechua y Mestico) no son homogéneos: los niños Aymará son más grandes que los niños de otros grupos rurales nativos de grandes alturas; esto es similar a lo que ocurre con los niños que viven en medio urbano a gran altitud. Es posible que la evolución secular acompañada de una mejor alimentación, en los años recientes, sean responsables de estos cambios.

El estudio comparativo de las estaturas de los grupos interétnicos indica que las poblaciones urbanizadas son ligeramente más altas que las poblaciones rurales y presentan también un grado de obesidad más marcado que sus homólogos rurales (Frisancho et al., 1970). Esta diferencia se detecta igualmente, entre las poblaciones amerindias de La Paz (Greksa et al.,1984).

Viviendo a alturas similares, las condiciones económicas más favorables parecieran tener efectos benéficos en el desarrollo más rápido del esqueleto del niño ( Pawson et al., 2001), de la talla y del peso; se observa también un nivel más alto de obesidad. Por otra parte, los individuos que viven a gran altitud tienen una circunferencia torácica más importante que aquellos que viven a altitudes más bajas (lannotti et al., 2009).

Si el desarrollo corporal de los niños es sensible al medio ambiente urbano, su origen social es también un parámetro que debe ser considerado. La talla media difiere según las categorías sociales: los niños provenientes de clases sociales acomodadas son en promedio, más grandes que los niños de clases modestas. Esta estratificación social no es excepcional (Stinson, 1982) y ha sido puesta en evidencia desde hace mucho tiempo por otros autores.

En el caso que nos concierne, las poblaciones de Quito, los resultados indican la existencia de una jerarquía de estaturas ligada al origen social del niño. Los niños de clase alta poseen una talla media superior y los niños de clase más modesta, una talla media más pequeña. Los niños de la clase media presentan resultados intermedios. Es conveniente señalar aquí, que incluso los niños pertenecientes a clases acomodadas los z-scores son inferiores a las normas de la OMS. Dittmar en 1997, señalaba que de acuerdo con las normas de la OMS, los niños de los dos géneros viviendo en altitud, independientemente de la etnia amerindia son de una estatura y peso inferior a los niños de USA de la misma edad.

Los factores que intervienen en el desarrollo corporal de los niños no son siempre numerosos. Si los miembros de la categoría social inferior poseen una talla mediana más pequeña que sus homólogos de otras categorías sociales, además del factor social, debe considerarse el origen geográfico de estos niños y de sus padres. Es así como, una proporción importante de niños y de padres son nativos de zonas rurales o de pequeños poblados de la Sierra, contrariamente a lo que sucede con otros grupos sociales.

La comparación con otras comunidades andinas es difícil de establecer puesto que la existencia en esos parajes condiciona la talla a la edad adulta. Estas diferencias se manifiestan durante el desarrollo (Crespo et al., 1995). La cordillera de los Andes y su gran desnivelación, dan lugar a la existencia, de una gran diversidad de ecosistemas.

Los factores no solamente medio ambientales, socioeconómicos e igualmente políticos pueden influenciar negativamente el desarrollo corporal de los niños (Pawson et al., 2010); también pueden conducir a un paro temporario de la evolución secular.

Los resultados obtenidos para la masa corporal (BMI) son claramente superiores a las normas de la OMS, independientemente de la categoría social o género. Tales resultados no permiten poner en evidencia problemas de desnutrición. Por el contrario, si se 
analizan los resultados de sobrepeso y de obesidad, para las niñas, ninguna diferencia se observa en función de las diversas clases sociales. En el caso de los niños, los grupos sociales se diferencian en la repartición de frecuencias correspondientes a las tasas de déficit ponderal y sobrepeso. Casi un cuarto de los niños pertenecientes a las categorías acomodadas y media padecen de sobrepeso. Esto representa el doble de lo que se detecta en los niños de clases modestas y a su vez, son opuestos a aquellos encontrados en los países industrializados, donde la más importante proporción de personas en situación de sobrepeso se hallan entre los miembros de las categorías sociales más desfavorecidas. Este fenómeno no se limita solamente a los países industrializados, es así como en México, alrededor de un tercio de los adolescentes y dos tercios de los adultos sufren de sobrepeso, entre 1999 y 2006 el sobrepeso ha aumentado de un tercio en los niños (Olaiz et al., 2006).

Esta diferencia en las tasas de sobrepeso registradas en las poblaciones modestas, puede encontrar una explicación en cuanto a su origen rural como ya lo señalaba Frisancho (1970), indicando que las poblaciones urbanizadas son ligeramente más grandes y considerablemente más obesas que sus homólogos rurales.

En los países industrializados, el predominio del sobrepeso e incluso de la obesidad, es inversamente proporcional al nivel de ingresos de la familia (Vigarello, 2010). La distribución de las tasas de sobrepeso y de obesidad, en los países industrializados muestran disparidades marcadas en función del nivel de educación y condiciones socioeconómicas, específicamente en el caso de las mujeres (Sassi et al., 2009). Actualmente, en Quito, pareciera que sean los miembros de las clases medias los más sensibles a la influencia de la publicidad de la sociedad de consumo, lo que explica el gran éxito de las bebidas azucaradas, las famosas "soft drink" (Popkin et al., 1998). Este medio ambiente favorece el consumismo y por ende la obesidad. La obesidad y el sobre peso al interior de las familias están ligados a la salud y en particular a la alimentación y a la actividad física.
La actividad física no está ligada exclusivamente a la práctica de un deporte, es así como en Quito los niños de clases media y acomodadas llegan a la escuela en autobús o en automóvil particular, por lo contrario, los niños de clases modestas se desplazan a pie para asistir a los centros de enseñanza.

En América Latina, las estratificaciones sociales son bien marcadas y generalmente son la resultante de las antiguas estructuras coloniales. Las costumbres alimenticias varían fuertemente según las categorías sociales. Una parte no despreciable de la población sufre siempre de malnutrición o de desnutrición a grados diversos. Los modos de restauración normalizados al estilo americano y los productos provenientes de la industria agroalimentaria, tienden remplazar los productos de la alimentación tradicional, ocasionando nuevas formas de carencias alimenticias, de sobrepeso y obesidad. Actualmente, este fenómeno alcanza sobre todo las grandes aglomeraciones (De Suremain et al., 2009).

Globalmente los cambios alimentarios no favorecen de ninguna manera a las categorías sociales pobres. Las humillaciones sociales conducen a un rechazo de los alimentos considerados un símbolo de los "pobres" y a la inversa, orientan las preferencias por los alimentos de los "ricos", los cuales no siempre son los más equilibrados desde un punto de vista nutritivo (Lentz, 1991). 


\section{Bibliografía}

Apfelbaun, M., Romon, M., Dubus, M., 2004. Diététique et nutrition. Paris, Masson.

Bejarano, I.F., Dipierri, J.E., Andrade, A., Alfaro,E.L., 2009. Geographic altitude, surnames, and height variation of Jujuy (Argentina) Conscrips. Am. J. Phys Antropol, $138: 158-163$.

Bennett, A. Sain, S.R., Vargas, E., Moore L.G., 2008. Evidence that parent-of-origin affects birth-weight réductions at high altitude. Am. J. Hum. Biol., 20(5) ; 592-597.

Benoist, J., 1980. Les Amériques. In Hiernaux, J. La diversité biologique humaine. Montréal, Presses Universitaires de Montréal/Paris, Masson, 341-393.

Cole, T.J., Bellizzi, M.C., Flegal, K.M., Dietz, W.H., 2000. Establishing a standard définition for children overweight and obesity. International Survey BMJ, 320 ; 1240-1243.

Cole, T.J., Flegal, K.M., Nicholls, D., Jackson, A., 2007. Body mass Index cut offs to define thinness in children and adolescents. International Survey. BMJ : 335 : 194.

Cossio-Bolaños, M.A., Arruda, M., Gómez Campos, R., 2009. Crecimiento físico en niños de 6 à 12 años de media altura de Arequipa - Perú (3220 msnm). Revista international de ciencas del deporte, $14: 32-44$.

Crespo.I., Valera, J. Gonzales G.F., Guerra-Garcia, R., 1995. Crecimiento y desarrollo de niños y adolescentes a diversas alturas sobre el nivel del mar. Acta Andina, 4(1): 53-64.

De Meer, K., Bergman, R., Kusner, J.S., Voorhoeve, H.W., 1993. Differences in physical growth of Ayamara and Quechua children living at high altitude in Peru. Am. J. Phys. Anthropol. 90(1):59-75.

De Onis, M., Onyango, A.W., Borghi, E., Siyam, B., Nishida, C., Siekmann, J., 2007. Development of WHO groxth reference for school-aged children and adolescents. Bulletin of the Worjd Health Organization, $85: 660-667$.

De Suremain, C.-E. , Katz, E., 2009. Introducción : Modelos alimentarios y recomposiciones sociales en America Latina. Anthropology of Food, S6, 1-14.
Dieu-Cambrézy, C. 1992. Croissance et adaptation physiologique de l'homme à l'altitude; une question d'actualité (Etat de Veracruz, Mexique). In: Bland-Pamard, C. (éd.) Dynamique des systèmes agraires; la santé en société. Regards et remèdes. Paris, ORSTOM, 251-272.

Dieu-Cambrezy, C., Froment, A., 1993. Croissance et hypoxie d'altitude dans la région du cofre de Perote (Etat de Veracruz, Mexique). Bull. et Mém. de la Société d'Anthropologie de Paris, T5, N³-4 : 401-416.

Dittmar, M., 1997. Linear growth in weight, stature, sitting height and leg lenght, and body proportions of Aymara school-children linving in an hypoxic environment at high altitude en Chile. $Z$. Morphol. Anthropol., 81(3): 333-344.

Eveleth, P.S., Tanner, J.M., 1976. Worldwide variation in human growth. Cambridge, Cambridge University Press.

Facchini, F., 2003. Les effets de l'altitude. In : Susanne., C. et al. Anthropologie biologique : Evolution et biologie humaine. Bruxelles De Boeck, 429-434.

FAO, 2003. Environment and natural resources. Working paper $n^{\circ} 10$, Rome, FAO. 